\section{IMPROVING HUMAN} AWARENESS DURING COLLABORATION WITH ROBOT: REVIEW

STEFAN GRUSHKO ${ }^{1}$, ALES VYSOCKY ${ }^{1}$, JIRI SUDER ${ }^{1}$, LADISLAV GLOGAR $^{2}$, ZDENKO BOBOVSKY ${ }^{1}$

${ }^{1}$ VSB - Technical University Ostrava, Faculty of Mechanical Engineering, Department of Robotics, Ostrava, Czech Republic

${ }^{2}$ AUTOKLASTR - Moravskoslezsky automobilovy klastr, z.s., Ostrava, Czech Republic

DOI: 10.17973/MMSJ.2021_12_2021181

e-mail : stefan.grushko@vsb.cz

Human-robot collaboration is a widespread topic within the concept of Industry 4.0. Such collaboration brings new opportunities to improve ergonomics and innovative options for manufacturing automation; however, most of the modern collaborative industrial applications are limited by the fact that neither collaborative side is fully aware of the partner: the human operator may not see the robot movement due to own engagement in the work process, and the collaborative robot simply has no means of knowing the position of the operator. Dynamic replanning of the robot trajectory with respect to the operator's current position can increase the efficiency and safety of cooperation since the robot will be able to avoid collisions and proceed in task completion; however, the other side of communication remains unresolved. This paper provides a review of methods of improving human awareness during collaboration with a robot. Covered techniques include graphical, acoustic and haptic feedback implementations. The work is focused on the practical applicability of the approaches, and analyses present challenges associated with each method.

KEYWORDS

Human-robot collaboration, awareness, interaction, notification, alerts, graphical interface, acoustic interface, haptic interface, virtual reality, mixed reality.

\section{INTRODUCTION}

Human-Robot Collaboration (HRC) is a promising trend in the field of industrial and service robotics. Collaborative robotics established new opportunities in the cooperation between humans and machines by enabling the robot to share the workspace with the personnel where it helps with nonergonomic, repetitive, uncomfortable, or even dangerous operations. With the growing level of cooperation, there is a tendency to increase the intertwining of human and robot workspaces, potentially leading to complete unification in the future (see Figure 1). By allowing a fully shared workspace for human workers and robots, it is possible to utilize the advantages of both, maximize the efficiency and minimize the time needed to complete a task. In HRC the main emphasis is placed on establishing safe work for the human operator [Vysocky and Novak, 2016]. On one side, the robot has to be aware of a human operator, and on the other side, the operator needs to be aware of the current status of the robot system. Advanced workplaces may include monitoring systems enabling the robot to react to and potentially predict [Mainprice and Berenson 2013, Hermann et al. 2015, Casalino et al. 2018] the operator's movements by immediately stopping the activity or by replanning the trajectory [Bolano et al. 2018]. Such workspace monitoring system enabling the robot to avoid collisions can be considered as one-side awareness; however, the other side of communication remains unresolved. Despite many efforts made to make robots understand and predict human actions, there is still a lack of communication from the robot to the human operator. Difficulties in understanding the robot's intent (planned trajectory, current task, internal status, error conditions) during demanding cooperation can lead to dangerous situations, reduced work efficiency and a general feeling of anxiety when working close to the robot (even if it is a collaborative robot). Moreover, in some cases, the position of the operator may be completely blocking the robot from performing the task, and the user's unawareness may lead to the lower overall productivity of the workspace or failed technological processes, for instance, when the robot task is strictly limited in time. Mutual understanding between human and robot is thus a fundamental aspect of achieving good efficiency and ergonomics in the task execution.

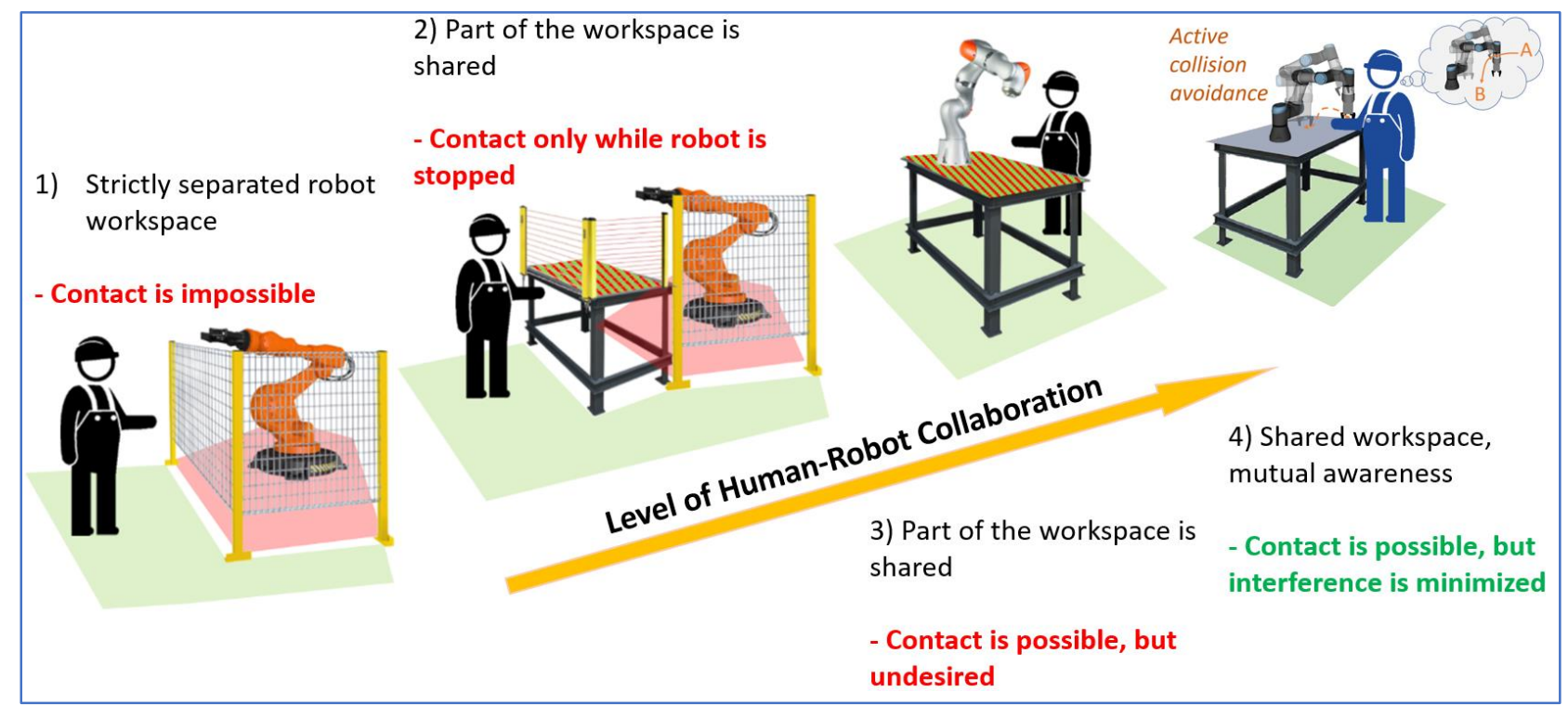

Figure 1. Illustration of the HRC levels: (1) - strictly separated robot workspace; (2) - partially shared workspace; (3) - fully shared workspace; (4) fully shared workspace with mutual awareness. Adapted from [Vysocky and Novak 2016] 
A possible solution is to provide intuitive communication channels that allow to inform the operator about the status of the robot, its current goal and warn the operator if the current activity blocks the robot from fulfilling its task. These communication channels may be realized with the help of feedback systems. To convey information, these systems may utilize the primary sensory modalities of a human: vision, hearing, touch.

\section{LITERATURE REVIEW}

This section will focus on analysing the state of the art in the field of improving operator's awareness during HRC in shared workspaces. The analysis will be focused on the practical applicability of the approaches and present challenges associated with each method. The covered approaches will be divided into groups according to the sensory modality it uses to convey information about the system to the user. Some of the referred studies have been targeting mobile robotics, however, their principles may also be applied to collaborative tasks with open-chain manipulators.

\subsection{Graphical feedback}

Many existing methods for communicating robot motion intent use graphical clues which notify the human worker about the status of the robot. Typical information for visualization may include the internal status of the robot, command acknowledgement, planned trajectory and current workspace borders. In the simplest example of such an approach, data visualizations are displayed on monitors (static or hand-held tablets), which, however, require the operator to interrupt the current task and check the visualization on display. Moreover, 2D displays can only provide a limited expression of the information and can be easily interfered with due to direct sunlight or obstructions. These limitations are significant because diverted attention may lead to violation of working procedures or even injury in certain tasks.

Light projectors represent a straightforward solution for visualizing additional graphical clues and notifications to the operator, possibly directly in the operator's vicinity, making it easier for the clues to be noticed [Andersen et al. 2016, Kalpagam et al. 2018]. In this approach, the light projectors can be placed stationary or mounted on a robot flange. A mounted projector can give the system a clear advantage over those that use only a stationary location, as it allows projections to be made almost anywhere in the vicinity (by moving the robot), even in hard-to-reach areas that cannot be covered by a stationary projector. [Reinhart et al. 2007] have demonstrated a spatial user interface implemented using a laser projector system placed on the robot flange, which provided the user with interactive visual clues projected to the workpiece. The projection system can monitor the working object and dynamically modify the visualized information. This allows, for instance, to notify the operator about the current workspace (Figure 2) utilized by the robot as well as the information about future action to be carried out by the robot. Projector-based systems have a number of limitations, the main one being that various obstacles and the operator can block the system from both displaying the graphical clues and tracking the work objects, leading to an increased risk of misinterpretation of the visualized information by the operator.

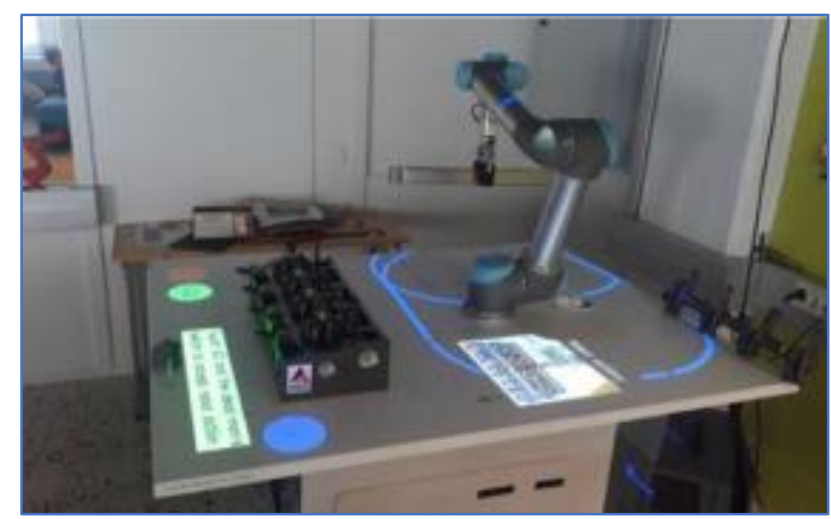

Figure 2. Depicting the robot work zone using a light projector (located above the worktable). Reproduced from [Hietanen et al. 2020], licensed under Creative Commons CC-BY-NC-ND

Multiple Augmented Reality (AR) and Mixed Reality (MR) approaches have been developed as a subsequent improvement of the projector-based solution that allows a more intuitive and immersive overlay of the visual notifications with the real environment as well as objects in the workspace. Augmented reality headsets allow 3D graphical clues to be displayed directly in the user's field of view without completely overlapping visual information from the real world [Bambusek et al. 2019]. For instance, the disadvantages of projector-based systems were addressed by projecting visual clues in augmented reality [Fang et al. 2014, Hietanen et al. 2020], which do not require the operator to interrupt his work and check the visualization on display. Visual clues may represent both the current workspace [Gkournelos et al. 2018, Hietanen et al. 2020] of the robot and its future trajectory - see Figure 3. Graphical visualization of robot trajectory may allow the worker to avoid robot interference and be more confident while working near the robot [De Pace et al. 2020].

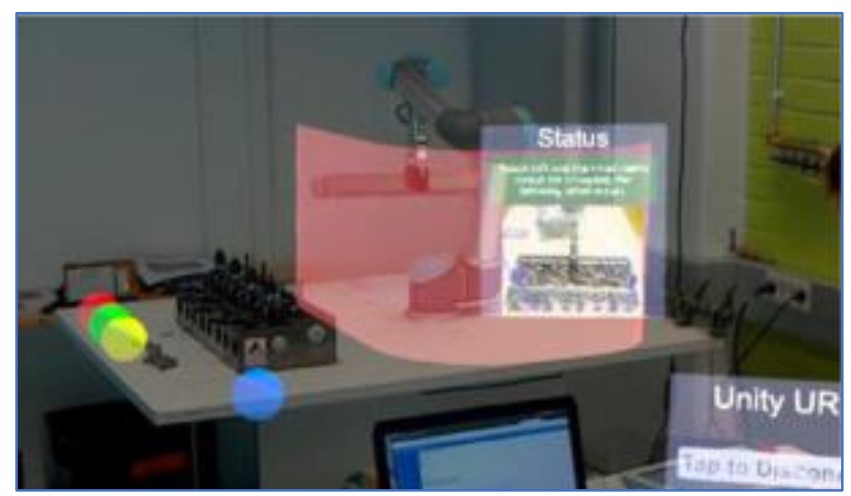

Figure 3. Projection of the robot's workspace to MR (Microsoft HoloLens). Reproduced from [Hietanen et al. 2020], licensed under Creative Commons CC-BY-NC-ND

The robot workspace may be visualized by displaying the whole robot body at each trajectory waypoint [Dietrich et al. 2010, Gadre et al. 2019] or by displaying only the trajectory of the endpoint. [Bolano et al. 2019, Bolano et al. 2021a] have demonstrated a system that combined MR graphical clues with speech interaction for implementing fluent collaboration with a collaborative robot. The developed system notifies the user about the space that the robot will occupy during its motion using MR representation of the swept volume of the planned robot motion. 
While the advances of AR and MR technologies have increased their usage in HRC applications, it is yet unclear how mature the wearable MR headset technology is for actual conditions of industrial manufacturing. One of the problems associated with the visualization of the planned movement of the robot and other visual information is that it cannot be guaranteed that this information is always in the field of view of the operator (the operator may be looking in a different direction). Moreover, when a large variety of robot data needs to be displayed, this may lead to the information overload of the human operator. Under such conditions, the operator will not be able to percept all the notifications provided by the system. It is also worth mentioning that MR devices themselves present an interfering component that may distract the operator during the task. Experimental user studies performed in [Hietanen et al. 2020] reported a comprehensive comparison and evaluation of HRC in a realistic manufacturing task in two conditions: a projectormirror setup and a wearable AR headset (Microsoft HoloLens). The results indicated that HoloLens was experienced less safe (comparing to the projector-based notification system) due to the intrusiveness of the device. Even though it was used as an augmented reality display, it blocked, to some extent, the view for the operator. Additionally, the device was considered bulky and ergonomically uncomfortable, as it created discomfort and decreased the feeling of safety during the task.

\subsection{Acoustic feedback}

Another option of improving the awareness of a human worker during HRC is by utilizing audio feedback. Auditory cues provide a wide range of contextual information that promotes awareness of a person about its surrounding. While vision feedback is traditionally preferred in applications that require a high level of accuracy, audio information is important in scenarios when other modalities that are limited, blocked, or noisy due to the circumstances. An example of an application of this approach was demonstrated in [Clair and Mataric 2015], where the efficiency of the collaborative task was enhanced by enabling the robot to use synthesised speech to give a human teammate acoustic feedback about the currently performed action. A study performed in [Cha et al. 2018] has explored the effects of different auditory signals on a human's ability to localize and predict the actions of a collaborating mobile robot. Another research [Bolano et al. 2018] implemented and verified a bidirectional acoustic interaction system, which has been integrated with a collaborative robotic environment. The system allowed the human operator to communicate with the robot using natural speech, whereas the robot was enabled to notify the user about its current actions and status. The researchers then extended the developed system [Bolano et al. 2021b] by combining graphical and acoustic feedback channels for user notification. In the designed shared workspace, the robot was aware of the position of the human operator and was able to replan its movements to avoid collisions. Graphical and acoustic (synthesised speech and specific action-sounds) feedback systems notified the operator about the robot's intentions and simplified the understanding of the robot's planned trajectories. The experiments conducted by the research group showed that the use of visual and acoustic feedback to give information related to the robot execution plan helped the operators to avoid discomfort while working close to the robot. Acoustic feedback may also be used as a channel of complementary information for the user, as well as a mean to ease the load on the visual channel. Study presented in [Liu et al. 2018] have presented multimodal interaction system which utilized multiple inputs from the user, such as speech commands, body and hand motions. The experiments conducted by the researchers shown that the proposed multimodal fusion model outperforms the three unimodal models. In general, however, speech recognition in industrial scenarios presents multiple challenges because the typical noisy environment can dramatically affect the interaction performance, leading to an unacceptable inaccuracy in understanding the uttered intention since the operator may be unable to discern the audio signals. Since the operator must be aware of its surrounding, the use of headphones is not an available solution. A possible solution for noisy environments may be introduced by the use of bone conduction [Dauman 2013] headphones, which enables better sound intelligibility.

\subsection{Haptic feedback}

Haptic feedback can be generally divided into two different classes:

- Kinaesthetic: senses generated by proprioceptors in the muscles, joints, tendons. Kinaesthetic feedback allows feeling performed movements, their speed, force resistance to the movement and joint positions [Grushko et al. 2020a].

- Tactile: senses generated by mechanoreceptors embedded in the skin. They enable the sensations of vibration, pressure, touch, pain.

Implementation of kinaesthetic feedback is generally more complicated as it requires stimulation of the proprioceptors. Only limited research was conducted in this field [Pfeiffer et al. 2019, Hasegawa et al. 2020] with typical implementation consisting of providing the user with force feedback enabled by functional electrical stimulation, which utilizes contraction of operator's muscles for activation of the proprioceptors in the agonist-antagonist muscle pairs. Unlike kinaesthetic feedback, implementation of tactile feedback is usually straightforward, and the foremost haptic interfaces use electrical actuators for providing it; examples of such actuators: vibration motors, solenoids, voice-coil actuators, piezo motors, SMA (ShapeMemory Alloy) muscles. Ubiquitous commercial products, including mobile phones, medical instruments, gaming controllers, and others, employ vibrations to transmit tactile notifications to their users. Vibration motors are suitable when the stimulation duration can be infinite and should be continuous since this is hardly achieved with the use of singleacting actuators such as SMA muscles (although an appropriate integration into the design may solve this limitation). Some studies [Pamungkas and Ward 2013, Sagardia et al. 2015] utilized electro-tactile stimulation as an alternative to mechanicallyprovided feedback. However, from the point of view of the utilization of senses by the human-machine interfaces, the haptic feedback often can be considered as underemployed. In activities requiring high attention, where both the visual and auditory channels are occupied because of the user involvement in the task or because blocked by the environment (production line noise), notifications delivered via visual and audio channels may fail to draw the user's attention. Recent studies focus on developing a new intuitive way of conveying information to users and off-loading part of the information to other human senses, specifically to the sense of touch. It represents a robust and direct way of transferring information to the user, making it suitable to convey information to workers in industrial 
environments, where visual and auditory modalities might be busy due to engaging in the task, or their efficiency may be diminished due to worn personal protective equipment. The most frequent applications for haptic feedback devices are providing the user with a better knowledge of the state of the system during teleoperation and programming. Study presented in [Barros et al. 2011] performed a set of tests using the simulation model of the teleoperated robot and enhancing the users with tactile feedback (using a compact hand-worn feedback device) that could notify them about the actual collisions of the robot with the surroundings. Another example of the use of a haptic feedback device is an implementation of a control interface for a swarm of quadcopters [Tsykunov et al. 2018] - the user was equipped with an interface glove that was simultaneously controlling the swarm and receiving the vibrotactile feedback that represented the current state of the swarm. Vibration devices can also be used during the programming of industrial robots by notifying the user about, for instance, approaching singularities and joint limits [Li et al. 2012, Sziebig and Korondi 2017] or commencing the next phase of the manufacturing process [Casalino et al. 2018]. A different approach was presented in [Grushko et al. 2021a], where handworn tactile feedback devices were utilized for communicating robot's motion intentions and its status to a human worker - see Figure 4.

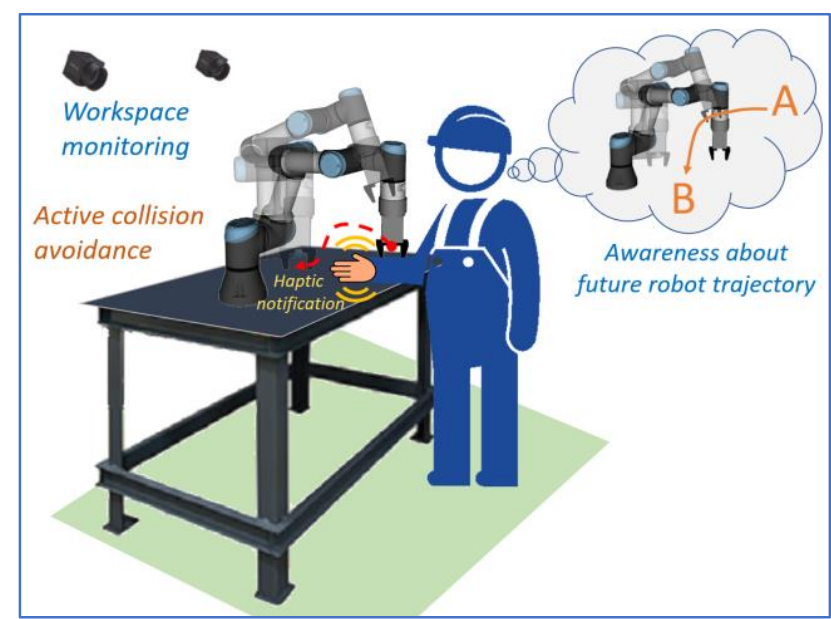

Figure 4. Principle of utilization of haptic notifications for informing the user about the future trajectory of the robot. Adapted from [Grushko et al. 2021b], licensed under CC BY 4.0

The presented collaborative system combined workspace monitoring, automatic robot's path replanning (based on optimized motion planning framework [Grushko et al. 2020b]) and haptic feedback devices, whose task was to reliably notify the human worker about the currently planned robot's trajectory. The closer the worker's hand approached the future segment of the trajectory, the stronger the vibration provided by the device (see Figure 5). The authors further extended the system and improved its intuitiveness for the user by implementing spatial tactile notifications where the intensity of the vibration of each tactor depended both on the relative position and orientation of the device and future trajectory of the robot [Grushko et al. 2021b]. The implemented system of spatially organized tactors is similar to tactile displays that are often utilized for assisting visually impaired people in tasks of spatial navigation accessing digital content, movement guiding, interaction with virtual objects.

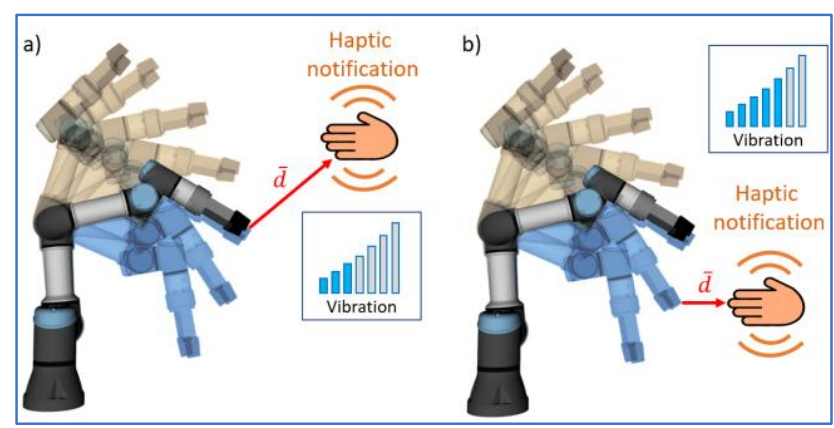

Figure 5. Illustrating distance notification principle: (a-b) vibration intensity is proportional to the distance to the closes point of the robot body in any timestep of the future trajectory. Adapted from [Grushko et al. 2021a], licensed under CC BY 4.0

The localisation system presented in the papers relied on the colour-based differentiation of the notification systems, which can be impractical in certain conditions. As an alternative, a commonly available stereovision-based hand tracking device, such as Leap Motion can be used [Vysocky et al. 2020]. It is also worth noting, that in certain cases, the executed task activity (such as handling an electric screwdriver) may block or distort the perception of the provided vibration alerts leading to higher chance of misinterpreting them.

A similar solution was implemented in [Scheggi et al. 2012, Scheggi et al. 2014], in which a mobile robot had the task of guiding a human (possibly sightless) from an initial to the desired target position through a cluttered corridor by only interacting with the human via bracelet with three embedded vibration motors. Research conducted in [Aggravi et al. 2016] presented a solution for guiding the hand of a human user using a vibrotactile haptic device placed on the user's forearm. In general, even though the tactile notifications represent a private communication channel (only the wearer of the feedback device can receive the notifications), they require a stable close contact with human skin in order to convey information.

\section{CONCLUSIONS}

This paper has provided an overview of existing approaches to improving human awareness during collaboration with the robot. Examples of implementation of the techniques as well as their shortcoming were provided and analysed. In general, it can be anticipated that combining multiple approaches will allow achieving more reliable information transfer to the user.

An important factor in any type of notification system is the way information is presented to the user. Provided notifications and prompts should be intuitive and unobtrusive over long periods of time. New notification strategies should be tested through a series of user studies to determine which ones are most intuitive for users. Finding the types of notifications and graphical prompts that are sufficiently visible and convenient for all users requires extensive research and multiple user studies, as they must be designed with both the individual user experience and security standards in mind. For example, in the case of vibrotactile notification, it is necessary to take into account the ISO 5349-1 standard, which defines the measurement and assessment of exposure to vibration transmitted through the hands, as well as the allowable exposure values. 
In an industrial setting, the described approaches may also be used not only to help to avoid the discomfort caused by unawareness about the intentions of the robot but also as a method to inform the user about the status of any device or technological process as well as danger present in the vicinity.

\section{ACKNOWLEDGMENTS}

This work was supported by the Research Platform focused on Industry 4.0 and Robotics in Ostrava Agglomeration project, project number CZ.02.1.01/0.0/0.0/17_049/0008425 within the Operational Program Research, Development, and Education.

\section{REFERENCES}

[Aggravi et al. 2016] Aggravi, M., Salvietti, G., Prattichizzo, D. (2016). Haptic wrist guidance using vibrations for Human-Robot teams. 2016 25th IEEE International Symposium on Robot and Human Interactive Communication (RO-MAN), 113-118. Available from https://doi.org/10.1109/ROMAN.2016.7745098.

[Andersen et al. 2016] Andersen, R. S., Madsen, O., Moeslund, T. B., Amor, H. B. (2016). Projecting robot intentions into human environments. 2016 25th IEEE International Symposium on Robot and Human Interactive Communication (RO-MAN), 294-301. Available from https://doi.org/10.1109/ROMAN.2016.7745145.

[Bambusek et al. 2019] Bambusek, D., Materna, Z., Kapinus, M., Beran, V., Smrz, P. (2019). Combining Interactive Spatial Augmented Reality with Head-Mounted Display for End-User Collaborative Robot Programming. 2019 28th IEEE International Conference on Robot and Human Interactive Communication (RO-MAN), 1-8. Available from https://doi.org/10.1109/ROMAN46459.2019.8956315.

[Barros et al. 2011] Barros, P. G. de, Lindeman, R. W., Ward, M. O. (2011). Enhancing robot teleoperator situation awareness and performance using vibro-tactile and graphical feedback. 2011 IEEE Symposium on 3D User Interfaces (3DUI), 47-54. Available from https://doi.org/10.1109/3DUI.2011.5759216.

[Bolano et al. 2021a] Bolano, G., Fu, Y., Roennau, A., Dillmann, R. (2021). Deploying Multi-Modal Communication Using Augmented Reality in a Shared Workspace. 2021 18th International Conference on Ubiquitous Robots (UR), 302-307. Available from https://doi.org/10.1109/UR52253.2021.9494689.

[Bolano et al. 2021b] Bolano, G., Iviani, L., Roennau, A., Dillmann, R. (2021). Design and Evaluation of a Framework for Reciprocal Speech Interaction in Human-Robot Collaboration. 2021 30th IEEE International Conference on Robot Human Interactive Communication (RO-MAN), 806-812. Available from https://doi.org/10.1109/ROMAN50785.2021.9515389.

[Bolano et al. 2019] Bolano, G., Juelg, C., Roennau, A., Dillmann, R. (2019). Transparent Robot Behavior Using Augmented Reality in Close Human-Robot Interaction (p. 7). Available from https://doi.org/10.1109/RO-MAN46459.2019.8956296

[Bolano et al. 2018] Bolano, G., Roennau, A., Dillmann, R. (2018). Transparent Robot Behavior by Adding Intuitive Visual and Acoustic Feedback to Motion Replanning. 2018 27th IEEE International Symposium on Robot and Human Interactive Communication (RO-MAN),
1075-1080

Available

https://doi.org/10.1109/ROMAN.2018.8525671.

[Casalino et al. 2018] Casalino, A., Messeri, C., Pozzi, M., Zanchettin, A. M., Rocco, P., Prattichizzo, D. (2018). Operator Awareness in Human-Robot Collaboration Through Wearable Vibrotactile Feedback. IEEE Robotics and Automation Letters, 3(4), 4289-4296. Available from https://doi.org/10.1109/LRA.2018.2865034.

[Cha et al. 2018] Cha, E., Fitter, N., Kim, Y., Fong, T., Mataric, M. (2018). Effects of Robot Sound on Auditory Localization in Human-Robot Collaboration (p. 442). Available from https://doi.org/10.1145/3171221.3171285.

[Clair and Mataric 2019] Clair, A. S., Mataric, M. (2015). How Robot Verbal Feedback Can Improve Team Performance in Human-Robot Task Collaborations. 2015 10th ACM/IEEE International Conference on Human-Robot Interaction (HRI), 213-220. Available from: https://doi.org/10.1145/2696454.2696491.

[Dauman 2013] Dauman, R. (2013). Bone conduction: An explanation for this phenomenon comprising complex mechanisms. European Annals of Otorhinolaryngology, Head and Neck Diseases, 130(4), 209-213. Available from https://doi.org/10.1016/j.anorl.2012.11.002.

[De Pace et al. 2020] De Pace, F., Manuri, F., Sanna, A., Fornaro, C. (2020). A systematic review of Augmented Reality interfaces for collaborative industrial robots. Computers Industrial Engineering, 149, 106806. Available from https://doi.org/10.1016/j.cie.2020.106806.

[Dietrich et al. 2010] Dietrich, A., Schulze, M., Zug, S., Kaiser, J. (2010). Visualization of robot's awareness and perception. In ACM International Conference Proceeding Series (p. 44). Available from https://doi.org/10.1145/1837154.1837160.

[Fang et al. 2014] Fang, H. C., Ong, S. K., Nee, A. Y. C. (2014). A novel augmented reality-based interface for robot path planning. International Journal on Interactive Design and Manufacturing (IJIDeM), 8(1), 33-42. Available from https://doi.org/10.1007/s12008-0130191-2.

[Gadre et al. 2019] Gadre, S. Y., Rosen, E., Chien, G., Phillips, E., Tellex, S., Konidaris, G. (2019). End-User Robot Programming Using Mixed Reality. 2019 International Conference on Robotics and Automation (ICRA), 2707-2713. Available from https://doi.org/10.1109/ICRA.2019.8793988

[Gkournelos et al. 2018] Gkournelos, C., Karagiannis, P., Kousi, N., Michalos, G., Koukas, S., Makris, S. (2018). Application of Wearable Devices for Supporting Operators in Human-Robot Cooperative Assembly Tasks. Procedia CIRP, 76, 177-182. Available from https://doi.org/10.1016/j.procir.2018.01.019.

[Grushko et al. 2020a] Grushko, S., Spurny, T., Cerny, M. (2020). Control Methods for Transradial Prostheses Based on Remnant Muscle Activity and Its Relationship with Proprioceptive Feedback. Sensors, 20(17), 4883. Available from https://doi.org/10.3390/s20174883.

[Grushko et al. 2020b] Grushko, S., Vysocky, A. Vyomkesh Kumar Jha, Pastor, R., Prada, E., Mikova, L. and Bobovsky, Z.. 2020. 'Tuning Perception and Motion Planning Parameters for Moveit! Framework'. https://doi.org/10.17973/MMSJ.2020_11_2020064. 
[Grushko et al. 2021a] Grushko, S., Vysocky, A., Oscadal, P., Vocetka, M., Novak, P., Bobovsky, Z. (2021). Improved Mutual Understanding for Human-Robot Collaboration: Combining Human-Aware Motion Planning with Haptic Feedback Devices for Communicating Planned Trajectory. Sensors, 21(11), $3673 . \quad$ Available from https://doi.org/10.3390/s21113673.

[Grushko et al. 2021b] Grushko, S., Vysocky, A., Heczko, D., Bobovsky, Z. (2021). Intuitive Spatial Tactile Feedback for Better Awareness about Robot Trajectory during Human-Robot Collaboration. Sensors, 21(17), 5748. Available from https://doi.org/10.3390/s21175748.

[Hasegawa et al. 2020] Hasegawa, Yuu, Tomoya Kitamura, Sho Sakaino, and Toshiaki Tsuji. 2020. 'Bilateral Control of Elbow and Shoulder Joints Using Functional Electrical Stimulation Between Humans and Robots'. IEEE Access 8: 15792-99. Available from https://doi.org/10.1109/ACCESS.2020.2967466.

[Hermann et al. 2015] Hermann, A., Mauch, F., Fischnaller, K., Klemm, S., Roennau, A., Dillmann, R. (2015). Anticipate your surroundings: Predictive collision detection between dynamic obstacles and planned robot trajectories on the GPU. 2015 European Conference on Mobile Robots (ECMR), 1-8. Available from https://doi.org/10.1109/ECMR.2015.7324047

[Hietanen et al. 2020] Hietanen, A., Pieters, R., Lanz, M., Latokartano, J., Kamarainen, J.-K. (2020). AR-based interaction for human-robot collaborative manufacturing. Robotics and Computer-Integrated Manufacturing, 63, 101891. Available from https://doi.org/10.1016/j.rcim.2019.101891.

[Kalpagam et al. 2018] Kalpagam G., R., Rathore, Y. K., Ross, H. M., Ben Amor, H. (2018). Better Teaming Through Visual Cues: How Projecting Imagery in a Workspace Can Improve Human-Robot Collaboration. IEEE Robotics Automation Magazine, 25(2), 59-71. Available https://doi.org/10.1109/MRA.2018.2815655

[Li et al. 2012] Li, H., Sarter, N. B., Sebok, A., Wickens, C. D. (2012). The Design and Evaluation of Visual and Tactile Warnings in Support of Space Teleoperation: Proceedings of the Human Factors and Ergonomics Society Annual Meeting. Available from https://doi.org/10.1177/1071181312561384.

[Liu et al. 2018] Liu, H., Fang, T., Zhou, T., Wang, L. (2018). Towards Robust Human-Robot Collaborative Manufacturing: Multimodal Fusion. IEEE Access, 6, 74762-74771. Available from https://doi.org/10.1109/ACCESS.2018.2884793.

[Mainprice and Berenson 2013] Mainprice, J., Berenson, D. (2013). Human-robot collaborative manipulation planning using early prediction of human motion. 2013 IEEE/RSJ International Conference on Intelligent Robots and Systems, 299-306. Available from https://doi.org/10.1109/IROS.2013.6696368.

[Pamungkas and Ward 2013] Pamungkas, Daniel S., and Koren Ward. 2013. 'Tele-Operation of a Robot Arm with Electro Tactile Feedback'. In 2013 IEEE/ASME International Conference on Advanced Intelligent Mechatronics, 704-9. Available from https://doi.org/10.1109/AIM.2013.6584175.

[Pfeiffer et al. 2019] Pfeiffer, Max, Samuel Navas Medrano, Jonas Auda, and Stefan Schneegass. 2019. 'STOP!
Enhancing Drone Gesture Interaction with Force Feedback'. In 1st International Workshop on HumanDrone Interaction. Glasgow, United Kingdom: Ecole Nationale de l'Aviation Civile [ENAC]. Available from https://hal.archives-ouvertes.fr/hal-02128395.

[Reinhart et al.] Reinhart, G., Vogl, W., Kresse, I. (2007). A Projection-based User Interface for Industrial Robots. 2007 IEEE Symposium on Virtual Environments, Human-Computer Interfaces and Measurement Systems. Available from https://doi.org/10.1109/VECIMS.2007.4373930.

[Sagardia et al. 2015] Sagardia, Mikel, Katharina Hertkorn, Thomas Hulin, Simon Schätzle, Robin Wolff, Johannes Hummel, Janki Dodiya, and Andreas Gerndt. 2015. 'VR-OOS: The DLR's Virtual Reality Simulator for Telerobotic on-Orbit Servicing with Haptic Feedback'. In 2015 IEEE Aerospace Conference, 1-17. Available from https://doi.org/10.1109/AERO.2015.7119040.

[Scheggi et al. 2012] Scheggi, S., Chinello, F., Prattichizzo, D. (2012). Vibrotactile haptic feedback for humanrobot interaction in leader-follower tasks. In ACM International Conference Proceeding Series (p. 4). Available https://doi.org/10.1145/2413097.2413161.

[Scheggi et al. 2014] Scheggi, S., Aggravi, M., Morbidi, F., Prattichizzo, D. (2014). Cooperative human-robot haptic navigation. In Proceedings-IEEE International Conference on Robotics and Automation. Available from https://doi.org/10.1109/ICRA.2014.6907245.

[Sziebig amd Korondi 2017] Sziebig, G., Korondi, P. (2017). Remote operation and assistance in human robot interactions with vibrotactile feedback. 2017 IEEE 26th International Symposium on Industrial Electronics (ISIE), 1753-1758. Available from https://doi.org/10.1109/ISIE.2017.8001513.

[Tsykunov et al. 2018] Tsykunov, E., Labazanova, L., Tleugazy, A., Tsetserukou, D. (2018). SwarmTouch: Tactile Interaction of Human with Impedance Controlled Swarm of Nano-Quadrotors. 2018 IEEE/RSJ International Conference on Intelligent Robots and Systems (IROS), 4204-4209. Available from https://doi.org/10.1109/IROS.2018.8594424.

[Vysocky and Novak 2016] Vysocky, A., Novak, P. (2016). Human-Robot collaboration in industry. $\mathrm{MM}$ Science Journal, 2016, 903-906. Available from https://doi.org/10.17973/MMSJ.2016_06_201611.

[Vysocky et al. 2020] Vysocka, A., Grushko, S., Oscadal, P., Kot, T., Babjak, J., Janos, R., Sukop, M., and Bobovsky, Z., 2020. 'Analysis of Precision and Stability of Hand Tracking with Leap Motion Sensor'. Sensors 20 (15): 4088. https://doi.org/10.3390/s20154088.

\section{CONTACTS:}

Ing. Stefan Grushko, Ph.D.

VSB - Technical University of Ostrava

Faculty of Mechanical Engineering, Department of Robotics, 17. Listopadu 2172/15, Ostrava, 708 33, Czech Republic +420 597329 443, stefan.grushko@vsb.cz, robot.vsb.cz 